\title{
Synthesis of Dibenzo[hi,st]ovalene and Its Amplified Spontaneous Emission in a Polystyrene Matrix
}

\author{
Giuseppe M. Paternò ${ }^{\ddagger},{ }^{[a]}$ Qiang Chen ${ }^{\ddagger},{ }^{[b]}$ Xiao-Ye Wang, ${ }^{[b]}$ Junzhi Liu, ${ }^{[c]}$ Silvia G. Motti, ${ }^{[a]}$ Annamaria \\ Petrozza, ${ }^{[a]}$ Xinliang Feng, ${ }^{[c]}$ Guglielmo Lanzani, ${ }^{[a, d]}$ Klaus Müllen* ${ }^{[b]}$ Akimitsu Narita ${ }^{*[b]}$ and \\ Francesco Scotognella ${ }^{*[a, d]}$
}

\begin{abstract}
A large number of graphene molecules, or large polycyclic aromatic hydrocarbons (PAHs), have been synthesized displaying varying optoelectronic properties. Nevertheless, their potential for application in photonics has remained largely unexplored. Herein, we describe the synthesis of a highly luminescent and stable graphene molecule DBO 1 with zigzag edges and elucidate its promising optical gain properties by means of ultrafast transient absorption spectroscopy. The incorporation of DBO 1 into an inert polystyrene matrix permits to observe amplified stimulated emission with a relatively low power threshold $\left(\approx 60 \mu \mathrm{J} \mathrm{cm}{ }^{-2}\right)$, thus highlighting its high potential for lasing applications.
\end{abstract}

Along with the development and successful commercialization of organic light-emitting diodes, extensive research has been devoted to the search for stable organic semiconductors (OSCs) that can be employed as optical gain media in laser devices ${ }^{[1]}$. The reasons for targeting organic lasers are: 1) the excellent optical features exhibited by organic luminescent materials, 2) the tunability of their optoelectronic properties by means of structural modulation and 3 ) the high solution processability ${ }^{[2]}$ that allows for the fabrication of low-cost devices. However, their considerable photodegradation upon intense laser irradiation and intrinsic instability against air and moisture are still major obstacles $^{[1 b]}$

Graphene materials have more recently emerged as promising alternative for stable luminescent systems. Although graphene itself has no bandgap, structural patterning into graphene nanostructures, i.e., nanographenes, such as quasione-dimensional graphene nanoribbons $(\mathrm{GNRs})^{[3]}$ and quasizero-dimensional graphene quantum dots (GQDs) ${ }^{[4]}$ allows to open a finite bandgap owing to quantum confinement of the electronic wave function. While top-down fabrication methods

[a] Dr. G. M. Paternò, S. G. Motti, Dr. A. Petrozza, Prof. G.Lanzan Center for Nano Science and Technology Istituto Italiano di Tecnologia

20133 Milano (Italy)

[b] Q. Chen, Dr. X.-Y. Wang, Prof. K. Müllen, Dr. A. Narita Max Planck Institute for Polymer Research

55128 Mainz (Germany)

email: muellen@mpip-mainz.mpg.de

email: narita@mpip-mainz.mpg.de

[c] Dr. J. Liu, Prof. X. Feng

Technische Universität Dresden, Center for Advancing Electronics

Dresden \& Department of Chemistry and Food Chemistry

01062 Dresden (Germany)

[d] Dr. F. Scotognella

Politecnico di Milano

Department of Physics

20133 Milano (Italy)

email: francesco.scotognella@polimi.it

[¥] These authors contributed equally to this work.

Supporting information for this article is given via a link at the end of the document. cannot precisely control the size and edge structure of the resulting nanographenes, bottom-up chemical synthesis from small molecular building blocks has proven to provide GNRs and GQDs, or graphene molecules, with atomically defined structures ${ }^{[5]}$. Such nanographene materials, indeed, exhibit intriguing luminescence features ${ }^{[6]}$ that depend strongly on the size and edge structures ${ }^{[7]}$, making them of great interest for plasmonic $^{[8]}$ and photonic applications ${ }^{[9]}$. There have been a few reports demonstrating or implying stimulated emission (SE) signal from structurally defined $\mathrm{GNRs}^{[10]}$ and graphene molecules $^{[6 a]}$ by transient absorption (TA) measurements in dispersions, which suggests their possible application as optical gain materials. However, to the best of our knowledge, there is hitherto no unambiguous proof for the SE from graphene molecules, and moreover actual amplified spontaneous emission (ASE) of such nanographene materials has never been observed in films. The exploration of ASE properties in nanographenes will provide a new class of stable materials as gain media in laser devices.

A variety of GNRs and graphene molecules featuring armchair-type edge structures have so far been synthesised ${ }^{[11]}$, but examples with extended zigzag edges are still limited, despite their intriguing properties in particular localized edge states or biradical ground-state characters ${ }^{[12]}$. Most of the graphene molecules with biradical ground states are highly unstable, hindering their in-depth characterisations and applications in devices ${ }^{[12 b, 13]}$. On the other hand, we have recently reported a "zigzag" HBC with four extra K-regions, forming two zigzag edges, which is highly stable and display intriguing optoelectronic properties with significantly lowered energy gap compared with the parent $\mathrm{HBC}^{[14]}$. Nevertheless, the "zigzag" HBC could not be obtained without iodo groups, which quenched its photoluminescence. Examples of such stable and low-energy-gap nanographene molecules with zigzag edges are still starkly limited.

In this work, we have synthesized dibenzo[hi,st]ovalene (DBO 1) (Scheme 1) as a novel and stable graphene molecule with both armchair and zigzag edges. DBO 1 exhibits a small optical gap (1.93 eV) based on the UV-vis absorption spectrum and an absolute photoluminescence quantum yield (PLQY) of as high as $79 \%$. The investigation of the excited state dynamics, carried out by means of resonant ultrafast TA spectroscopy, clearly reveals the occurrence of SE transitions in solution. Although the prominent optical properties of DBO 1 were largely quenched in the solid state, we were able to recover the SE signal by blending the molecule with polystyrene (PS), which, interestingly, led to the observation of ASE action from a $1 \mathrm{w} \%$ DBO 1 : PS composite film.

The synthesis of DBO 1 was carried out as displayed in Scheme 1. First, Suzuki coupling of 4-dodecyl-2(trimethylsilylethynyl)phenylboronic acid (2) and 7-bromo-2naphthaldehyde

(3) gave 
(trimethylsilylethynyl)phenyl)-2-naphthaldehyde (4) in 97\% yield. Then CuCl-mediated Glaser coupling of $\mathbf{4}$ provided diaryldiacetylene $\mathbf{5}$ in $98 \%$ yield. Subsequently, the key intermediate, bischrysene dialdehyde 6 was obtained through a $\mathrm{PtCl}_{2}$-catalysed cycloaromatization in $48 \%$ yield. Direct cyclodehydrogenation of dialdehyde $\mathbf{6}$ to $\mathbf{9}$ under various Scholl conditions failed, most probably because of the strong electron withdrawing properties of the two aldehyde groups. Therefore, compound 6 was reduced with $\mathrm{NaBH}_{4}$ and then protected with acetyl groups to obtain 7 in $86 \%$ yield over two steps. Notably, the oxidative cyclodehydrogenation of 7 succeeded with 2,3 dichloro-5,6-dicyano-1,4-benzoquinone

(DDQ)/trifluoromethanesulfonic acid $\left(\mathrm{CF}_{3} \mathrm{SO}_{3} \mathrm{H}\right)$ at a low temperature $\left(-78{ }^{\circ} \mathrm{C}\right)$, providing cove-edged graphene molecule 8 in 33\% yield. The low temperature was crucial for this conversion, and no product was obtained when the reaction was carried out at $0{ }^{\circ} \mathrm{C}$. The ester groups of 8 were then transformed to dialdehyde groups through a hydrolysis/oxidation sequence in $70 \%$ yield. To convert the cove edges of 9 into zigzag edges, 9 was treated with mesitylmagnesium bromide to give a diol intermediate, which reacted with $\mathrm{BF}_{3} \cdot \mathrm{OEt}_{2}$ under air to afford the target DBO 1 as a blue solid in $24 \%$ yield. The structure of DBO 1 was unambiguously proven by spectroscopic analysis. Fig. 1a displays the MALDI-TOF MS spectrum of 1 with an intense signal at $m / z=1044.66 \mathrm{Da}$, consistent with the expected molecular mass of $1044.66 \mathrm{Da}$. The experimental isotopic distribution was in good agreement with the calculated spectrum based on the chemical composition of $\mathrm{C}_{80} \mathrm{H}_{84}$. A well-resolved ${ }^{1} \mathrm{H}$ NMR spectrum of DBO 1 could be recorded in toluene- $d_{8}$ at $100{ }^{\circ} \mathrm{C}$ (Fig. 1b), and all the proton signals could be assigned with the assistance of 2D NMR spectroscopy (see SI).

The electrochemical property of DBO 1 was studied by cyclic voltammetry (CV) in a dichloromethane solution with ferrocene as an external standard (Fig. S2). Two reversible oxidation and reduction waves were observed, indicating the stability of these redox species. The HOMO and LUMO energy levels were estimated to be $-4.84 \mathrm{eV}$ and $-3.22 \mathrm{eV}$, based on the onset oxidation and reduction potentials, respectively, with the electrochemical HOMO-LUMO gap of $1.62 \mathrm{eV}$.

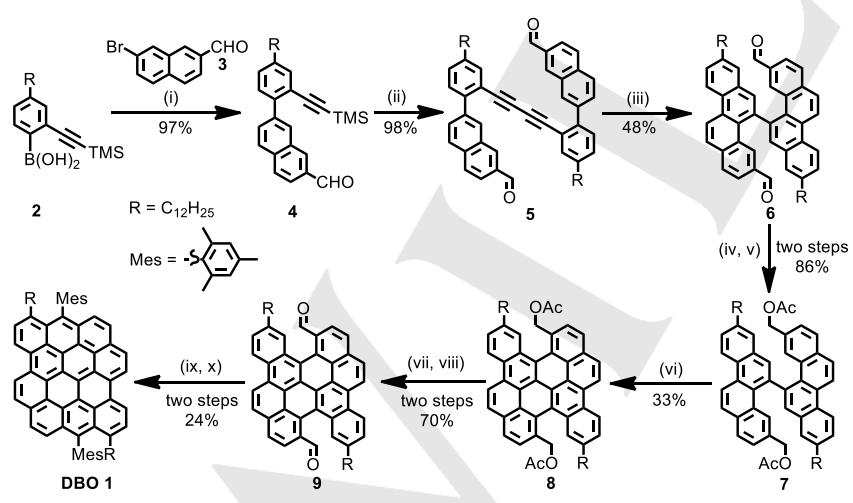

Scheme 1. Reaction conditions: (i) $\mathrm{Pd}\left(\mathrm{PPh}_{3}\right)_{4}$ (0.1 eq.), $\mathrm{Na}_{2} \mathrm{CO}_{3}$ (6.0 eq.) toluene/EtOH $/ \mathrm{H}_{2} \mathrm{O}=4: 1: 1,80{ }^{\circ} \mathrm{C}, 6 \mathrm{~h}$; (ii) $\mathrm{CuCl}\left(1.0\right.$ eq.), DMF, $80{ }^{\circ} \mathrm{C}, 12 \mathrm{~h}$ under air; (iii) $\mathrm{PtCl}_{2}$ (0.3 eq.), toluene, $80{ }^{\circ} \mathrm{C}, 48$ h; (iv) $\mathrm{NaBH}_{4}$ (4.0 eq.), $\mathrm{THF} / \mathrm{MeOH}=2: 1$, r.t., $2 \mathrm{~h}$; (v) Acetic anhydride, TEA (3.0 eq.), DMAP (0.3 eq.), $\mathrm{DCM}$, r.t., $2 \mathrm{~h}$; (vi) $\mathrm{DDQ}$ (4.0 eq.), $\mathrm{DCM} / \mathrm{CF}_{3} \mathrm{SO}_{3} \mathrm{H}=20: 1,-78{ }^{\circ} \mathrm{C}, 2 \mathrm{~h}$; (vii) $\mathrm{KOH}, \mathrm{THF} / \mathrm{EtOH} / \mathrm{H}_{2} \mathrm{O}, 80{ }^{\circ} \mathrm{C}, 12 \mathrm{~h}$; (viii) PCC (2.0 eq.), DCM, r.t., 2 h; (ix) MesMgBr (20.0 eq.), THF, r.t. 2 h; (x) $\mathrm{BF}_{3} \cdot \mathrm{OEt}_{2}$, DCM, r.t., 2 h, under air. DMF dimethylformamide; THF: tetrahydrofuran; TEA: triethylamine; DMAP: 4dimethylaminopyridine; DCM: dichloromethane; PCC: pyridinium chlorochromate.
The UV-vis absorption spectrum of DBO 1 in a toluene solution showed a maximum at $625 \mathrm{~nm}$ with a large absorption coefficient of $1.83 \times 10^{5} \mathrm{M}^{-1} \mathrm{~cm}^{-1}$ (Fig. 2a). This absorption band could be assigned to HOMO $\rightarrow$ LUMO transition based on timedependent density functional theory (TD-DFT) calculations at the B3LYP/6-311G(d,p) level (see Fig. S1 and Table S1). In comparison to the spectrum of precursor 9, the low-energy absorption band was red-shifted by $88 \mathrm{~nm}$ due to the formation of zigzag edges and extension of aromatic core. The optical gap decreased from $2.19 \mathrm{eV}$ to $1.93 \mathrm{eV}$ upon the conversion of $\mathbf{9}$ into 1 , according to the onsets of their UV-vis absorption spectra. The emission spectra of $\mathbf{9}$ and $\mathbf{1}$ in toluene solutions displayed maxima at 561 and $637 \mathrm{~nm}$, with small Stokes shifts of 23 and $12 \mathrm{~nm}$, respectively, indicating the rigid structures of these two molecules. Interestingly, the emission of 1 could be observed already under room light excitation (Fig. 2b), which is a clear signature of its strong emission properties. The absolute PLQY amounts to $79 \%$ (average value $=66.9 \%$ with a standard deviation of $6.6 \%$ over 35 measurements). To the best of our knowledge, this is one of the highest values reported for graphene molecules ${ }^{[15]}$. These promising photophysical features highlight the important role of the zigzag edges in DBO $\mathbf{1}$, significantly lowering the optical gap of $\mathbf{9}$ and realizing highly efficient red light emission.

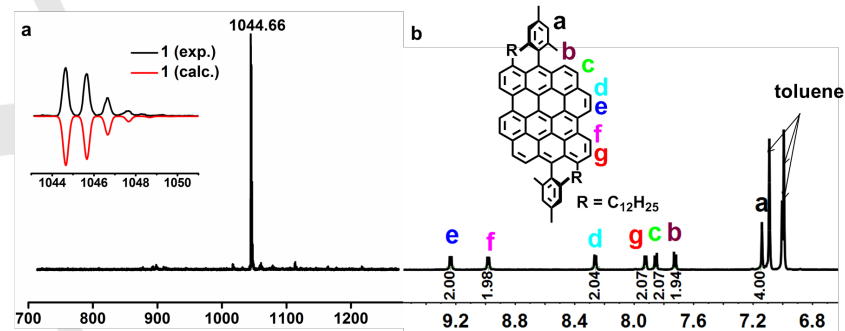

Figure 1. a) MALDI-TOF mass spectrum of DBO 1; Inset shows a comparison between the calculated and experimental isotopic distributions; b) Aromatic region of the ${ }^{1} \mathrm{H}$ NMR spectrum of DBO 1 , recorded in toluene- $\mathrm{d}_{8}$ at $100{ }^{\circ} \mathrm{C}$ (700 MHz).
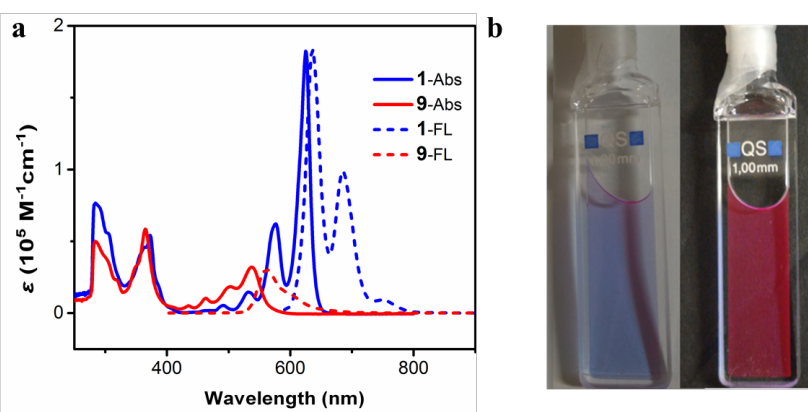

Figure 2. a) UV-vis absorption and fluorescence spectra of precursor 9 and DBO $1\left(10^{-5} \mathrm{M}\right.$ in toluene for all measurements). b) Photographs of a toluene solution of DBO 1 with (right) and without (left) a black background, showing the red luminescence under room light.

The high PLQY, sharp absorption and emission peaks and small Stokes shift observed for DBO 1 prompted us to gain a deeper insight into its photophysics ${ }^{[16]}$. We carried out broadband $(375-800 \mathrm{~nm})$ TA measurements by using a pump pulse of $625 \mathrm{~nm}$ that is in resonance with the HOMO $\rightarrow$ LUMO transition.. The transient spectrum of DBO 1 (Fig. 3) displays 
four features, namely: (i) a negative signal centred at $450 \mathrm{~nm}$ that can be interpreted as a photoinduced absorption (PA) from the first excited state $S_{1}$ to higher excited states $S_{i}$; (ii) a positive signal at $650 \mathrm{~nm}$ that can be assigned to the depletion of the ground state due to the main $\mathrm{HOMO} \rightarrow$ LUMO transition (photobleaching, PB); (iii) another positive PB signal centred at $570 \mathrm{~nm}$, corresponding to its vibronic replica and (iv) a positive signal at $695 \mathrm{~nm}$. The latter is a sign of stimulated emission given that: $i$ ) it is not present in the steady state absorption and ii) is red-shifted with respect to the main HOMO $\rightarrow$ LUMO transition peak $^{[16 a]}$. Although the origin of the SE signal in such graphene molecule is currently under investigation, we suppose it is related to vibronic relaxation within the higher-lying electronic excited state.

Stimulated emission signals are of great interest for acquiring direct information about possible optical gain properties of the material. Thus, we focus the discussion mainly on the SE signal and report the complete transient spectra and time-dynamics (up to $1 \mathrm{~ns}$ ) data sets in the supplementary information. From the transient spectra of DBO 1 in toluene solution (Fig. 3a, top graph) the degree of decay is relatively low for all the signals (Fig. S3a-b). This is indicative of stable excited states that can sustain stimulated emission and can also explain the high PLQY ${ }^{[17]}$. In addition, the sharp rise of all the transient signals, along with their linear dependence on pump fluence (see Fig. S3c-d), suggest that the photoexcitation mechanism is direct and does not involve any non-linear process.

For comparison, we have also performed ultrafast TA studies of soluble hexa-peri-hexabenzocoronene (HBC) derivative $^{[18]}$, which has solely the armchair edge structure, in toluene solution. The preliminary data (Fig. S4) show that all the transient features are incorporated in a large photoinduced absorption signal even at lower concentrations (down to 0.01 $\mathrm{mg} / \mathrm{mL}$ ). Furthermore, we could not observe any clear SE signal in the probed region (from $430 \mathrm{~nm}$ to $650 \mathrm{~nm}$ ) for this HBC derivative, pointing to the uniqueness of the SE observed for DBO 1 and implying an important role of the zigzag edge for achieving the optical gain properties in graphene molecules.

We next turn to the characterisation of DBO 1 in thin film, which is essential for device applications (Fig. 3a, central graph). It appears that the transient peaks are broader than those in solution (e.g., for the PB signal: $F W H M_{\text {solution }}=19.4 \pm 0.4 \mathrm{~nm}$; $\mathrm{FWHM}_{\text {film }}=34.3 \pm 0.9 \mathrm{~nm}$ ), and all the transient signal intensities decrease considerably in $\approx 200 \mathrm{fs}$ (Fig. S5a-b). Thereby, the SE peak is overwhelmed by a negative PA feature in a time-frame that is comparable with the instrumental resolution (Fig. 3b). The broadening of the peaks points toward the aggregation of graphene molecules in films ${ }^{[6 a]}$. The latter effect can be indicative of ultrafast and non-radiative intermolecular charge transfer processes in the solid state, which are promoted by the

effective supramolecular packing occurring in such planar molecules ${ }^{[19]}$, resulting in complete quenching of the $\mathrm{PL}$ in films.

To exploit gain properties of the material and possibly employ these features in solid-state devices, we embedded DBO 1 into a polystyrene (PS) matrix by simply mixing their solutions in toluene with $10,5,3$ and $1 \mathrm{w} \%$ ratios. Such dilution approach has been used to inhibit intermolecular charge-transfer processes, and thus enhance SE signals ${ }^{[19 a, 20]}$ and ASE/LASER action in luminescent conjugated polymers ${ }^{[21]}$. Notably, this method works effectively also for graphene molecules, as blending allows one to extend the SE signal of DBO 1 for about $\approx 70 \mathrm{ps}$ in the $1 \mathrm{w} \% 1$ :PS blend (Fig. 3b; see Fig. S6a-h for the spectra and dynamics of all the DBO 1:PS weight ratios). Moreover, the PS blending permits recovery of the emission of DBO 1 in the solid state, and a photoluminescence from the $1 \mathrm{w} \%$ DBO 1:PS composite film can be detected upon UV-light exposure at $365 \mathrm{~nm}$ (Fig. 3c). It is worth noting that by diluting the graphene molecule DBO 1 in the PS matrix, the transient spectral features start resembling those observed in solution, with a progressive narrowing of the $\mathrm{PB}$ peaks, and a suppression of the PA signal (Fig. 3a and Fig. S6). All these spectroscopic findings point toward the occurrence of excitations with charge-transfer character, as it has been reported for luminescent conjugated polymers ${ }^{[16 a, 19 a, 20,22]}$. In particular, both PA bands at $450 \mathrm{~nm}$ and $750 \mathrm{~nm}$ seem to stem from the photoinduced excitations of charges possibly originated upon intermolecular transfer, as their intensities decrease in solution and in blends with PS.
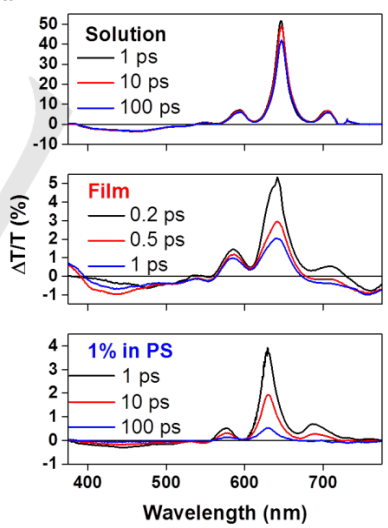

Figure 3. a) Ultrafast transient spectra of DBO 1 in solution $(0.05 \mathrm{mg} / \mathrm{mL}$ in toluene), film and $1 \mathrm{w} \%$ blend with PS (deposited on fused silica substrates by drop-casting); b) Normalised time-decay dynamics for the SE emission signal (probe at $695 \mathrm{~nm}$ ) for solution, film and $1 \mathrm{w} \%$ blend with PS. The spectra and time-decay dynamics shown above were taken with a pump fluence of $\approx 50 \mu \mathrm{J}$ $\mathrm{cm}^{-2}$; c) Comparison of the luminescence of DBO 1 in solution and $1 \mathrm{w} \%$ PS composite film under UV irradiation at $365 \mathrm{~nm}$. 
b

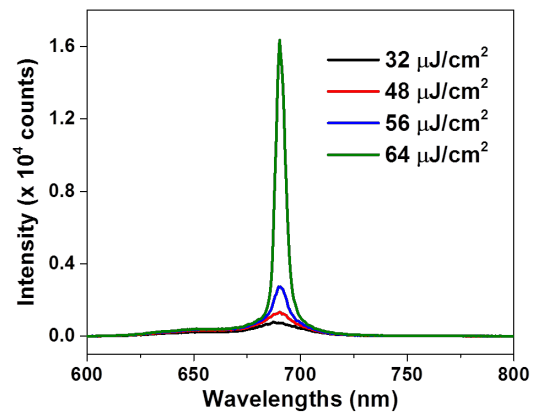

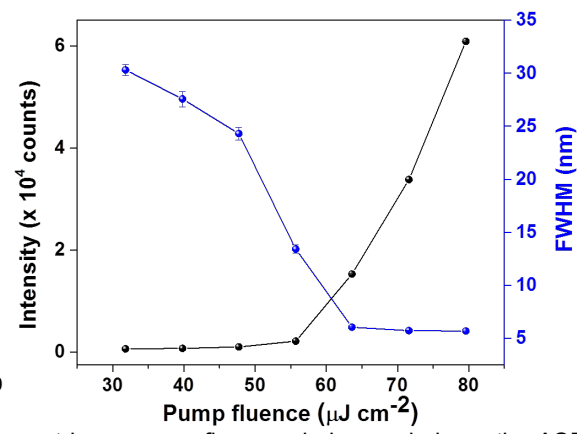

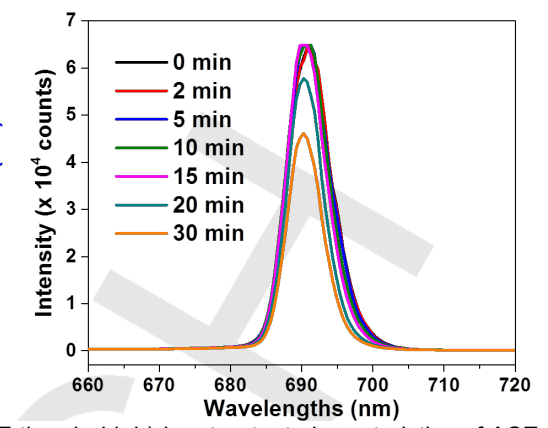

Figure 4. a) Photoluminescence spectra taken at laser power fluences below and above the ASE threshold; b) input-output characteristics of ASE action for the $1 \mathrm{w} \%$ blend in PS; c) evolution of the ASE signal over time taken at a laser pump fluence 5 times higher than the ASE threshold (320 $\left.\mu \mathrm{J} \mathrm{cm}^{-2}\right)$.

Remarkably, the $1 \mathrm{w} \%$ DBO 1:PS blend demonstrates amplified stimulated emission (ASE) action triggered above a relatively low laser fluence of $60 \mu \mathrm{J} \mathrm{cm}^{-2}$ (Fig. 4a-b). Although $\mathrm{PL}$ line narrowing is observed also in the $3 \mathrm{w} \%$ blend (Fig. S7ab), the laser power needed for FWHM narrowing is higher than the one required for the $1 \mathrm{w} \%$ blend $\left(\approx 150 \mu \mathrm{J} \mathrm{cm} \mathrm{cm}^{-2}\right)$. This suggests that the $1 \mathrm{w} \%$ blend can minimize the intermolecular cross talking (in terms of charge transfer) that is crucial for avoiding fast non-radiative deactivations, and promote ASE action. Although the $10 \mathrm{w} \%$ and $5 \mathrm{w} \%$ blends do not show any ASE action, they still allow to observe a PL signal (Fig. S7c-d). To test the stability of DBO 1 against photodegradation, the sample was irradiated under air at a laser fluence 5 times higher than the ASE threshold $\left(320 \mu \mathrm{J} \mathrm{cm}^{-2}\right)$, yielding only a $30 \%$ decay after 30 minutes of intense irradiation $\left(2 \times 10^{6}\right.$ pulses $)$. Although DBO 1 : PS blend films were stored under air, the same ASE emission was observed at least for 6 months after the preparation. The remarkable stability of DBO 1 against air, moisture and photodegradation must be ascribed to, both, the intrinsic robustness of its excited states, and to its incorporation into the inert PS matrix.

In summary, we have successfully synthesized DBO 1 as a novel graphene molecule with a unique combination of armchair and zigzag edges, demonstrating a low energy gap and strong red emission with remarkable PLQY of $79 \%$ as well as promising optical gain properties with low ASE threshold $\left(\approx 60 \mu \mathrm{J} \mathrm{cm}^{-2}\right)$ and high photochemical stability. With good solubility and thus enhanced processability, DBO 1 holds great potential for future applications in low-cost organic devices, such as LASERs and OLEDs. Moreover, this study demonstrates the promise of graphene molecules with zigzag edges as stable and highly luminescent materials with optical gain properties. Further variety of such graphene molecules are presently synthesized in our laboratories and studied in view of their role as optical gain materials.

\section{Acknowledgements}

We thank the financial support from Graphene Flagship, the Max Planck Society, the European Union Project MoQuaS, and the EU Horizon 2020 Research and Innovation Programme under Grant Agreement N. 643238 (SYNCHRONICS) and N. 642196 (iSwitch). X.-Y. W. is grateful for the fellowship from Alexander von Humboldt Foundation.
Keywords: graphene molecule• zigzag edges • transient absorption • stimulated emission • amplified spontaneous emission

[1] a) I. D. Samuel, G. A. Turnbull, Chem. Rev. 2007, 107, 1272-1295; b) A J. Kuehne, M. C. Gather, Chem. Rev. 2016, 116, 12823-12864; c) W. Zhang, J. Yao, Y. S. Zhao, Acc. Chem. Res. 2016, 49, 1691-1700; d) C. Kallinger, M. Hilmer, A. Haugeneder, M. Perner, W. Spirkl, U. Lemmer, J. Feldmann, U. Scherf, K. Müllen, A. Gombert, V. Wittwer, Adv. Mater. 1998, 10, 920-923; e) N. Tessler, Adv.Mater. 1999, 11,363-370.

[2] S. R. Forrest, Nature 2004, 428, 911-918.

[3] a) A. Narita, X. Feng, Y. Hernandez, S. A. Jensen, M. Bonn, H. Yang, I. A. Verzhbitskiy, C. Casiraghi, M. R. Hansen, A. H. Koch, G. Fytas, O. Ivasenko, B. Li, K. S. Mali, T. Balandina, S. Mahesh, S. De Feyter, K. Mullen, Nat.Chem. 2014, 6, 126-132; b) Y. W. Son, M. L. Cohen, S. G. Louie, Nature 2006, 444, 347-349.

[4] a) B. Trauzettel, D. V. Bulaev, D. Loss, G. Burkard, Nat. Phys. 2007, 3 , 192-196; b) L. Cao, M. J. Meziani, S. Sahu, Y. P. Sun, Acc. Chem. Res. 2013, 46, 171-180.

[5] a) A. Narita, X. Y. Wang, X. Feng, K. Müllen, Chem. Soc. Rev. 2015, 44 6616-6643; b) L. J. Zhi, K. Müllen, J. Mater. Chem. 2008, 18, 1472 1484; c) Y. Segawa, H. Ito, K. Itami, Nat. Rev. Mater. 2016, l, 15002.

[6] a) S. Zhu, L. Wang, B. Li, Y. Song, X. Zhao, G. Zhang, S. Zhang, S. Lu, J. Zhang, H. Wang, H. Sun, B. Yang, Carbon 2014, 77, 462-472; b) S. Zhu, Q. Meng, L. Wang, J. Zhang, Y. Song, H. Jin, K. Zhang, H. Sun, H. Wang, B. Yang, Angew. Chem. 2013, 52, 3953-3957.

[7] K. A. Ritter, J. W. Lyding, Nat. Mater. 2009, 8, 235-242.

[8] J. D. Cox, F. Javier Garcia de Abajo, Nat. Commun . 2014, 5, 5725.

[9] a) V. Gupta, N. Chaudhary, R. Srivastava, G. D. Sharma, R. Bhardwaj, S Chand, J. Am. Chem. Soc. 2011, 133, 9960-9963; b) S. Osella, A. Narita, M. G. Schwab, Y. Hernandez, X. Feng, K. Mullen, D. Beljonne, ACS Nano 2012, 6, 5539-5548.

[10] G. Soavi, S. Dal Conte, C. Manzoni, D. Viola, A. Narita, Y. Hu, X. Feng, U. Hohenester, E. Molinari, D. Prezzi, K. Mullen, G. Cerullo, Nat Commun. 2016, 7, 11010.

[11] a) X. Yang, X. Dou, A. Rouhanipour, L. Zhi, H. J. Rader, K. Müllen, $J$. Am. Chem. Soc. 2008, 130, 4216-4217; b) M. G. Schwab, A. Narita, Y. Hernandez, T. Balandina, K. S. Mali, S. De Feyter, X. Feng, K. Mullen, J. Am. Chem. Soc. 2012, 134, 18169-18172; c) M. El Gemayel, A. Narita, L. F. Dossel, R. S. Sundaram, A. Kiersnowski, W. Pisula, M. R. Hansen, A. C. Ferrari, E. Orgiu, X. Feng, K. Mullen, P. Samori, Nanoscale 2014, 6, 6301-6314.

[12] a) P. Ruffieux, S. Wang, B. Yang, C. Sanchez-Sanchez, J. Liu, T. Dienel, L. Talirz, P. Shinde, C. A. Pignedoli, D. Passerone, T. Dumslaff, X. Feng, K. Mullen, R. Fasel, Nature 2016, 531, 489-492; b) A. Konishi, Y. Hirao, K. Matsumoto, H. Kurata, R. Kishi, Y. Shigeta, M. Nakano, K. Tokunaga, K. Kamada, T. Kubo, J. Am. Chem. Soc. 2013, 135, 1430-1437.

[13] J. Liu, P. Ravat, M. Wagner, M. Baumgarten, X. Feng, K. Müllen, Angew. Chem. 2015, 54, 12442-12446.

[14] T. Dumslaff, B. Yang, A. Maghsoumi, G. Velpula, K. S. Mali, C. Castiglioni, S. De Feyter, M. Tommasini, A. Narita, X. Feng, K. Mullen, J. Am. Chem. Soc. 2016, 138, 4726-4729.

[15] a) S. Seifert, K. Shoyama, D. Schmidt, F. Würthner, Angew.Chem. 2016 55, 6390-6395; b) S. Müller, K. Müllen, Chem. Commun. 2005, 4045$55,6390$.

[16] a) J. Cabanillas-Gonzalez, G. Grancini, G. Lanzani, Adv. Mater. 2011, 23, 5468-5485; b) G. Cerullo, C. Manzoni, L. Luer, D. Polli, Photochem.\& Photobiol. Sci. 2007, 6, 135-144.

[17] J. R. Lakowicz, Principles of fluorescence spectroscopy, Springer Science \& Business Media, 2013.

[18] M. Kastler, W. Pisula, D. Wasserfallen, T. Pakula, K. Mullen, J. Am Chem. Soc. 2005, 127, 4286-4296.

[19] a) M. Yan, L. J. Rothberg, E. W. Kwock, T. M. Miller, Phys. Rev. Lett. 1995, 75, 1992-1995; b) N. J. Turro, Modern molecular photochemistry, University science books, 1991. 
WILEY-VCH

[20] T. Virgili, D. Marinotto, C. Manzoni, G. Cerullo, G. Lanzani, Phys. Rev. Lett. 2005, 94, 117402.

[21] M. Morales-Vidal, P. G. Boj, J. M. Villalvilla, J. A. Quintana, Q. Yan, N. T. Lin, X. Zhu, N. Ruangsupapichat, J. Casado, H. Tsuji, E. Nakamura, M. A. Diaz-Garcia, Nat Commun. 2015, 6, 8458. [22] T. Virgili, D. Marinotto, G. Lanzani, D. D. C. Bradley, Appl.Phys.Lett.
$\mathbf{2 0 0 5}, 86,91113$. 